\title{
Association of Mycotoxin and Sclerotia Production with Compatibility Groups in Aspergillus flavus from Peanut in Argentina
}

\author{
M. Victoria Novas and Daniel Cabral, Depto. de Cs. Biologicas, FCEN, Universidad de Buenos Aires, Ciudad \\ Universitaria, 1428 EHA, Buenos Aires, Argentina
}

\begin{abstract}
Novas, M. V., and Cabral, D. 2002. Association of mycotoxin and sclerotia production with compatibility groups in Aspergillus flavus from peanut in Argentina. Plant Dis. 86:215-219.

Vegetative compatibility (VC) of Aspergillus flavus isolates from peanut seed was studied to evaluate preliminary diversity and its association with mycotoxin production and sclerotia production and number. A. parasiticus isolates also were included as a comparative group. Isolates were divided into five categories based on mycotoxin production combination. Five of the $A$. flavus isolates were considered atypical because they simultaneously produced aflatoxins $\mathrm{B}, \mathrm{G}$, and cyclopiazonic acid (CPA). Vegetative compatibility groups (VCGs) were determined through complementation tests between nitrate-nonutilizing mutants. Sclerotia diameters and the number of sclerotia produced per square centimeter were determined for each isolate. Out of 32 isolates of A. flavus, 25 combined in 13 VCGs, whereas the remaining could not be assigned to any particular group. Each VCG included isolates of the same mycotoxin category, with only one exception. Also, all isolates within the same VCG were characterized by their ability to produce or not produce sclerotia. Isolates between VCGs showed significant differences in number of sclerotia per square centimeter, but differences in sclerotia size were not evident. Atypical isolates simultaneously producing aflatoxins B, G, and CPA formed a single and exclusive VCG.
\end{abstract}

Aflatoxins are carcinogenic secondary metabolites that occur in foods and feeds worldwide (3). Aspergillus flavus Link:Fr., A. parasiticus Speare, and A. nomius Kurtzman, Horn, \& Hesseltine produce a number of aflatoxins in agricultural commodities such as peanut seed $(15,22,36)$. Furthermore, A. flavus frequently produces cyclopiazonic acid (CPA; 17,26). Conventional methods for the prevention of aflatoxin contamination are not reliable on a commercial scale for any of the crops affected (11). There is interest in developing a biological control agent that can increase crop safety by decreasing aflatoxin content. Such a method may depend on the displacement of toxigenic isolates using atoxigenic isolates of the same species. However, before the employment of a mycotoxin biological control, it is necessary to study the genetic diversity of the population of interest. Toxicological studies on CPA have shown that it appears to act primarily as an entero-nephrotoxin in chickens and pigs and as a hepatotoxin in

Corresponding author: D. Cabral

E-mail: cabral@bg.fcen.uba.ar

This research was supported by PRHIDED-CONICET (Publication no. 145) and University of Buenos Aires.

Accepted for publication 27 September 2001.

Publication no. D-2002-0103-01R

(C) 2002 The American Phytopathological Society rats (17). A. flavus produces B aflatoxins $\left(\mathrm{B}_{1}\right.$ and $\left.\mathrm{B}_{2}\right), \mathrm{CPA}$, both, or none of these toxins $(22,33,36)$. A parasiticus typically produces, in addition to $\mathrm{B}$ aflatoxins, aflatoxins $\mathrm{G}_{1}$ and $\mathrm{G}_{2}$, but not CPA. A. nomius exhibits the same mycotoxin pattern as $A$. parasiticus, but morphologically resembles A. flavus. However, "atypical" isolates of A. flavus simultaneously producing B and $\mathrm{G}$ aflatoxins have been reported $(4,16$, 26,36).

A. flavus isolates that produce sclerotia can be divided into two types of strains, $\mathrm{L}$ and $\mathrm{S}$, on the basis of physiological, morphological, and genetic criteria $(3,10)$. S strain isolates produce numerous small sclerotia $(<400 \mu \mathrm{m}$ in diameter) and fewer conidia than $\mathrm{L}$ strain isolates. Typical isolates (L) of A. flavus may produce only B aflatoxins or no aflatoxins at all and all $\mathrm{S}$ strain isolates (named atypical isolates by Saito et al.; 34) reported to date produce large quantities of aflatoxins, some producing only B aflatoxins and others producing both $\mathrm{B}$ and $\mathrm{G}$ aflatoxins. Relationships among A. flavus isolates were inferred from restriction site variability in a portion of the Taka amylase A gene (18). In this study, two isolates that simultaneously produced $B$ and $G$ aflatoxins (21) were determined as intermediate between $A$. flavus and $A$. parasiticus by a phenetic analysis and as ancestral to both species by a cladistic analysis (18).

A. flavus has no sexual stage; however, some of its variability can be examined by sorting isolates into vegetative compatibil- ity groups (VCGs). VCGs have been associated with many morphological and physiological features, such as sclerotia size, mycotoxin production, and spatial distribution, in this genus $(1,23,32)$. Heterokaryon formation can only occur between strains with identical alleles at each of several loci governing vegetative compatibility (19,31). Aspergillus strains produce no clear signs of anastomosis or antagonism when they meet as wild strains; therefore, complementary nitrate-nonutilizing (nit) mutants are commonly used to identify compatible isolates $(1,32)$. Once identified, complementary mutants are paired. Complementation is indicated by a zone of dense, wild-type growth and heavy sporulation at the intersection of the two colonies due to hyphal anastomosis and nutritional complementation in the heterokaryon. Heterokaryons can form only between nit mutants of vegetative compatible isolates. Thus, isolates that are vegetative compatible belong to the same VCG.

In Argentina, 99\% of peanut production is localized in the center of Córdoba province. Isolates of A. flavus and A. parasiticus were previously obtained from peanut seed in this region to examine their mycotoxin production (36). Five isolates identified as A. flavus by their morphological characteristics simultaneously produced aflatoxins B and $\mathrm{G}$ and also CPA as reported by Blaney et al. (4). Each of the isolates also produced sclerotia.

The objectives of this preliminary study were to (i) determine for the first time in Argentina and in South America VCG diversity in A. flavus isolates from peanut seed that may enable a future development of a biocontrol strategy and (ii) evaluate any relationship between VCGs and mycotoxin production and sclerotia size and number, mainly between typical and atypical isolates.

\section{MATERIALS AND METHODS}

Isolates. Isolates of A. flavus $(n=32)$ and $A$. parasiticus $(n=11)$ had been obtained from peanut seed from 19 peanut fields in Córdoba province, Argentina, in a previous study (36). Categories into which Aspergillus isolates were placed (Table 1), based on toxin production, have been modified from Vaamonde et al. (37). Once the present study was finished, all "atypical" isolates and one isolate per VCG were placed in the Culture Collection, Facultad de Ciencias Exactas y Naturales (BAFC 
cultures) of the University of Buenos Aires (Table 2).

VCGs. The nit mutants of $A$. flavus and A. parasiticus were produced and their phenotypes were determined by the methods developed by Cove (12) and modified by Bayman and Cotty (2). Plugs of mycelia of Aspergillus isolates were transferred from potato dextrose agar (PDA) to Czapek-Dox $(\mathrm{Cz})$ plates containing potassium chlorate at $25 \mathrm{~g} /$ liter with nonadjusted $\mathrm{pH}$. Cultures were incubated at $30^{\circ} \mathrm{C}$ and the margins of the colonies with restricted growth were examined regularly for fastgrowing sectors, consisting of sparse my-

Table 1. Characterization of Aspergillus flavus and A. parasiticus isolates on the basis of mycotoxin production combination

\begin{tabular}{lccc}
\hline $\begin{array}{l}\text { Species, } \\
\text { category }\end{array}$ & $\begin{array}{c}\text { Aflatoxin } \\
\text { B }\end{array}$ & $\begin{array}{c}\text { Aflatoxin } \\
\text { G }\end{array}$ & CPA $^{\mathbf{z}}$ \\
\hline A. flavus & & & \\
I & + & - & + \\
II & + & + & + \\
III & + & - & - \\
IV & - & - & + \\
A. parasiticus & & & \\
V & + & + & - \\
\hline
\end{tabular}

${ }^{\mathrm{z}}$ Cyclopiazonic acid production. celium. Aspergillus nit mutants grow as wild-type isolates in $\mathrm{Cz}$ containing ammonium; therefore, hyphal tips from fastgrowing sectors were transferred to plates of $\mathrm{Cz}$ in which ammonium, added as ammonium tartrate, was used instead of $\mathrm{NaNO}_{3}$. The nit mutant phenotypes were determined by growing them on several media amended with different nitrogen sources (12). Three classes of mutants were recovered: niaD (nitrate nonutilizing), nirA (nitrite and nitrate nonutilizing), and cnx (hypoxanthine and nitrate nonutilizing). At least two different nit mutants were obtained from each strain. The source of nitrogen in the selection medium influenced the ratio of mutant classes (2). In A. flavus isolates, nirA mutants appeared frequently on glutamate (2); therefore, when it was not possible to obtain at least two different mutants from an isolate, we replaced the chlorate with this medium. To test for complementation, pairs of complementary mutants were inoculated $15 \mathrm{~mm}$ apart in the center of $\mathrm{Cz}$ plates and incubated for 7 to 14 days at $30^{\circ} \mathrm{C}$. Compatibility was identified by a line of wild-type growth at the zone of interaction. Initially, complementary pairings of niaD, nirA, and cnx mutants within each isolate were made to assess self-compatibility. Isolates that did not demonstrate self-compatibility were excluded from the analyses. For each isolate, an niaD, an $n i r A$, and, when available, a $c n x$ were selected as testers for pairing with new nit mutants. To establish VCGs, testers were paired in all possible combinations. As each group of new nit mutants was generated, they were paired against all testers for VCGs recognized at that time. All positive reactions were repeated for verification.

Sclerotial production. For sclerotia production, plates ( 60 by $15 \mathrm{~mm}$ ) containing $\mathrm{Cz}$ were inoculated with mycelia. Cultures were incubated in darkness at $30^{\circ} \mathrm{C}$ for 14 days (23). Sclerotia were obtained by scraping the surface of the plate (three replicate plates per isolate) over a no. 2 Whatman filter paper during irrigation with water containing Tween 20 (100 $\mu \mathrm{l} /$ liter), followed by rinsing with running tap water. Sclerotia were further cleaned in a beaker with repeated rinses and decanting, and later were air dried.

When an isolate, formerly identified as a sclerotia producer, did not produce any sclerotia under the culture conditions of this study, we cultured it in the same media used for the identification, malt extract

Table 2. Aspergillus flavus and A. parasiticus vegetative compatibility group (VCG) and isolate characteristics ${ }^{\mathrm{u}}$

\begin{tabular}{|c|c|c|c|c|c|}
\hline Species, culture ${ }^{v}$ & VCG & Category & Sclerotia mean $^{w}$ & Sclerotium diameter $(\mu \mathrm{m}) \pm$ SD $^{\mathbf{x}}$ & Strain isolate ${ }^{y}$ \\
\hline \multicolumn{6}{|l|}{ A. flavus } \\
\hline M35N1A & A & IV & None & None & $\ldots$ \\
\hline M34N2 & A & IV & None & None & $\ldots$ \\
\hline M15N3 & A & I & None & None & $\ldots$ \\
\hline M11N1/544z & A & IV & None & None & $\ldots$ \\
\hline M11N1X & A & IV & None & None & $\ldots$ \\
\hline M12N1 & B & I & $15.78 \mathrm{a}$ & $435 \pm 103 \mathrm{e}$ & $\mathrm{L}$ \\
\hline M6N1/ 117z & B & I & $20.4 \mathrm{a}$ & $345.23 \pm 80.99 \mathrm{~d}$ & $\mathrm{~L}$ \\
\hline M32N1 & B & I & $17 \mathrm{a}$ & $640.88 \pm 189.94 \mathrm{~g}$ & $\mathrm{~L}$ \\
\hline M38N1 & B & I & $6.6 \mathrm{a}$ & $453.44 \pm 114.07 \mathrm{e}$ & $\mathrm{L}$ \\
\hline M29N1 & B & I & $7.1 \mathrm{a}$ & $423 \pm 97.8 \mathrm{e}$ & $\mathrm{L}$ \\
\hline $\mathrm{M} 32 \mathrm{~N} 4 / 490^{\mathrm{z}}$ & $\mathrm{C}$ & II & $207.8 \mathrm{c}$ & $297.8 \pm 70.9 \mathrm{c}$ & $\mathrm{S}$ \\
\hline $\mathrm{M} 38 \mathrm{~N} 3 / 657^{\mathrm{z}}$ & $\mathrm{C}$ & II & $242.6 \mathrm{~d}$ & $215.71 \pm 76.7 \mathrm{a}$ & $\mathrm{S}$ \\
\hline $\mathrm{M} 35 \mathrm{~N} 2 / 589^{\mathrm{z}}$ & $\mathrm{C}$ & II & $230.3 \mathrm{~cd}$ & $282.4 \pm 53.4 \mathrm{bc}$ & $\mathrm{S}$ \\
\hline M36N1/294z & $\mathrm{D}$ & I & $2 \mathrm{a}$ & $636.2 \pm 153.9 \mathrm{~g}$ & $\mathrm{~L}$ \\
\hline M3N1X & $\mathrm{D}$ & I & $11 \mathrm{a}$ & $520.8 \pm 242.14 \mathrm{f}$ & $\mathrm{L}$ \\
\hline $\mathrm{M} 22 \mathrm{~N} 3 / 172^{\mathrm{z}}$ & E & IV & $134.8 \mathrm{~b}$ & $258 \pm 65.9 \mathrm{~b}$ & $\mathrm{~S}$ \\
\hline M36N2 & $\mathrm{E}$ & IV & $155.3 \mathrm{~b}$ & $299 \pm 70.03 c$ & $\mathrm{~S}$ \\
\hline M2N1 & $\mathrm{F}$ & I & 14.8 & $552.6 \pm 113.2$ & $\mathrm{~L}$ \\
\hline M10A & $\mathrm{G}$ & I & 10.4 & $580.2 \pm 128.67$ & $\mathrm{~L}$ \\
\hline M10N3 & $\mathrm{H}$ & I & 11.3 & $584.4 \pm 128.4$ & $\mathrm{~L}$ \\
\hline M11N3 & I & I & None & None & $\ldots$ \\
\hline M14N1 & $\mathrm{J}$ & I & None & None & $\ldots$ \\
\hline M14N2 & $\mathrm{K}$ & I & None & None & $\ldots$ \\
\hline M29N3 & $\mathrm{L}$ & III & None & None & $\ldots$ \\
\hline M40N1 & M & IV & 201.1 & $265.11 \pm 73.2$ & $\mathrm{~S}$ \\
\hline \multicolumn{6}{|l|}{ A. parasiticus } \\
\hline M15N1 & $\mathrm{N}$ & $\mathrm{V}$ & $\ldots$ & $\ldots$ & $\ldots$ \\
\hline $\mathrm{M} 15 \mathrm{~N} 2 / 611^{\mathrm{z}}$ & $\mathrm{N}$ & $\mathrm{V}$ & $\ldots$ & $\ldots$ & $\ldots$ \\
\hline M3N1/617z & $\mathrm{O}$ & V & $\ldots$ & $\ldots$ & $\ldots$ \\
\hline M12N3 & $\mathrm{O}$ & V & $\ldots$ & $\ldots$ & $\ldots$ \\
\hline M25N1 & $\mathrm{P}$ & $\mathrm{V}$ & $\ldots$ & $\ldots$ & $\ldots$ \\
\hline $\mathrm{M} 25 \mathrm{~N} 3$ & Q & $\mathrm{V}$ & $\ldots$ & $\ldots$ & $\ldots$ \\
\hline
\end{tabular}

u None = no sclerotia were produced. Only VCGs with two or more isolates were statistically analyzed. Means in a column not sharing a common letter are significantly different $(P<0.05)$ according to a least significant difference test.

${ }^{\vee}$ Aspergillus sp. and culture designation.

${ }^{\mathrm{w}}$ Mean per square centimeter, based on three square centimeters, chosen randomly, from each of three replicate plates per isolate on Czapek-Dox.

${ }^{x}$ Means based on 26 sclerotia, chosen randomly, from each of three replicates plates per isolate.

${ }^{\mathrm{y}}$ Strain $\mathrm{S}$ isolates produce sclerotia that average $<400 \mu \mathrm{m}$ in diameter; strain L isolates produce sclerotia $>400 \mu \mathrm{m}$ in diameter.

${ }^{\mathrm{z}}$ BAFC cult: Culture Collection number, Facultad de Ciencias Exactas y Naturales, Universidad de Buenos Aires, Argentina. 
agar (MEA). We also incubated this isolate on peanut seed previously autoclaved with distilled water. If all these procedures failed, we considered that this isolate had lost its ability to produce sclerotia.

Sclerotial size and number. To estimate diameters and number of sclerotia, three replicate observations were made per isolate using a gridline. A grid of lines was marked on the bottom of a 6-by-15-mm dish to form squares of $1 \mathrm{~cm}^{2}$ each. Samples were observed under a stereomicroscope. To assess diameters, sclerotia were spread out on the petri dish and measurements of 26 sclerotia per replicate, which intersected a line that had been previously chosen randomly, were recorded. It was assumed that sclerotium shape was approximately a prolate spheroid. Numbers of sclerotia were enumerated by counting three squares of $1 \mathrm{~cm}^{2}$ each, chosen randomly, from each replicate.

Data analysis. Only VCGs with two or more isolates were statistically analyzed. Sclerotia data (number and diameter) were analyzed using an unbalanced hierarchically two level nested analysis of variance design, followed by least significant difference mean separation tests $(P=0.05)$. The software used was the Statistical Package BIOM (F. James Rohlf) 1982.

\section{RESULTS}

nit Mutants. Most isolates formed two or three complementary mutants. When we replaced $\mathrm{KClO}_{3}$ with glutamate to increase the frequency of nirA mutants, we did not obtain the expected results. Out of 39 isolates, from which we recovered at least two types of nit mutants, 31 were selfcompatible.

We obtained many niaD mutants, one or two nirA of each isolate, and we also recovered one cnx in seven of these isolates. Isolates were paired according to complementation tests to study self-compatibility. As previously mentioned, compatibility was identified by a line of wild-type growth at the zone of interaction. A positive reaction between two different mutants types of isolate M15N2 is shown in Figure 1A. Most of the isolate pairings failed to generate the heterokaryon; therefore, we chose only the nit mutants that showed a positive reaction as testers of the isolates.

In some cases, we noticed that pairings previously defined as positive turned out negatively and that it was coincident with a phenotype change in one of the testers, expressed as an increase in sporulation and aerial mycelia.

VCGs. Complementation tests revealed 13 VCGs among the $32 \mathrm{~A}$. flavus isolates named A to $\mathrm{M}$. The largest VCGs (A and B) included five isolates each, one included three (C), four VCGs had two isolates (D and $\mathrm{E}$ ), and the last eight were represented by a single isolate $(\mathrm{F}-\mathrm{M}$; Table 2). A. parasiticus isolates grouped into four VCGs, two composed by two isolates each
( $\mathrm{N}$ and $\mathrm{O}$ ) and two represented by single isolates ( $\mathrm{P}$ and $\mathrm{Q}$; Table 2). All isolates that would be expected to complement with each other, based on interactions with a third isolate, did complement; however, we did test all possible combinations. None of the VCGs included isolates of the two species used in this study, A. flavus and $A$. parasiticus.

Eight isolates were self-incompatible; therefore, they were excluded from the analyses. The negative reaction between isolates M35N1B (self-incompatible) and $\mathrm{M} 11 \mathrm{~N} 1$ is shown in Figure 1B.

Some pairs of isolates produced positive and negative reactions in different combinations of nit mutants.

All of the isolates grouped in the same VCG produced the same combination of mycotoxins and sclerotia production, with the exception of M15N3, which differed in the combination of mycotoxins (Table 2).

As previously mentioned, atypical isolates M11N2 and M5N9 were excluded from the analyses. The other three isolates were vegetatively compatible with each other and vegetatively incompatible with the rest of the isolates, so we placed them in the same VCG $(\mathrm{C})$.

We determined VCG diversity of A. fla$v u s$, expressed as the number of groups divided by the total number of isolates (22), obtaining an index of 0.52 .

Most isolates placed in the same VCG had been collected from different fields and those fields were distant from each other.

Sclerotial production. In a previous study (36), 22 isolates of $A$. flavus were identified as sclerotial producers. All isolates produced sclerotia under our culture conditions, except for isolate M4N1. When we cultured this isolate on MEA and on peanut seed, no sclerotia were produced.

All isolates produced some sclerotia with diameters $>400 \mu \mathrm{m}$; therefore, they could be classified as L strain isolates by the Cotty criterion (10). However, the average was $<400 \mu \mathrm{m}$ in some isolates (Table 2).

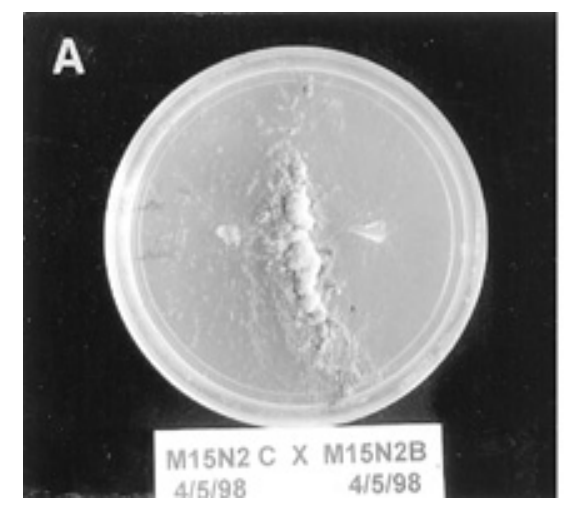

Fig. 1. Complementation among nitrate-nonutilizing (nit) mutants of Aspergillus flavus. As a result of complementation, dense hyphal growth occurred between two different nit mutants of A, M15 N1 isolate (self-compatible isolate), and $\mathbf{B}$, no complementation is observed between M35N1B and M11N1 isolates.
Analyses of variance showed significant differences among VCGs $(P<0.0001)$ in number of sclerotia per square centimeter and in sclerotia size. However, isolates within a VCG were significantly different in sclerotia size $(P>0.0001)$ but not in number of sclerotia per square centimeter $(P=0.334)$ (Table 2).

\section{DISCUSSION}

In the present study, A. flavus VCG diversity from peanut seed and its correlation with mycotoxin and sclerotia production was determined for the first time in Argentina and in South America.

As expected, none of the VCGs included isolates of the two species studied. As has been stated (22), although A. flavus and $A$. parasiticus are closely related based on their DNA complementation $(28,29)$, the lack of any vegetative compatibility between these species suggests that they are genetically isolated from each other.

We obtained approximately $90 \%$ of niaD, $8 \%$ of nirA, and $2 \%$ of cnx mutants. This proportion of different mutant types matches results obtained in other studies. The relationship found by Horn and Greene (22) for the three mutants types was niaD > nirA > cnx. Bayman and Cotty (2) detected 80 to $90 \%$ of niaD, $10 \%$ of nirA, and $10 \%$ of cnx mutants. Most mutants of the same isolate failed to complement with each other; also, some pairs of isolates produced a positive and negative reaction in different combinations of nit mutants. These results are similar to those of Brooker et al. (6), who observed that each isolate also sectored mutants that were unable to complement with any of the other mutants derived from the same isolate. In addition, some mutants could complement with only some of the other compatible isolates. Results obtained in nirA mutants of three isolates (data not shown) indicate that these mutants lack the ability to form heterokaryons. It had been suggested that the use of mutants could introduce the possibility of a negative pairing reaction as a result of other noncomple-

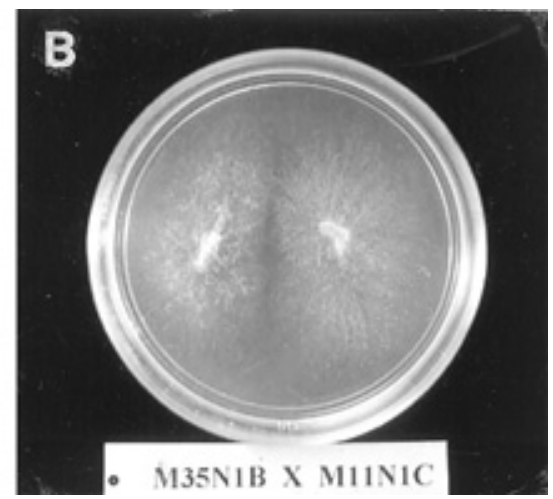


mentary mutations (20). Therefore, we paired the isolates in all possible combinations with each other to avoid a false negative.

Some isolates were self-incompatible and, therefore, could not be assigned to any VCG. Heterokaryon self-incompatibility (HSI) isolates already have been noticed in A. flavus $(22,32)$ and also in other species, such as Fusarium oxysporum $(8,25), F$. moniliforme (9), Colletotrichum spp. (6), Rhizoctonia solani (24), Verticillium alboatrum (7), and V. lecanii (27). The explanation of this phenomenon is still uncertain $(9,32)$.

The high A. flavus diversity index that we obtained (0.52), agrees with the results of other studies $(1,22)$. Horn et al. (23) proposed that the high VCG diversity of $A$. flavus in corn (32), cottonseed (1), and peanut (22) is similar to nonpathogenic soil isolates of $F$. oxysporum (20). In contrast, many populations of $F$. oxysporum pathogenic to plants have low VCG diversities due to the selective advantage of some VCGs in infecting a specific host $(5,30)$. Therefore, they suggested that none of the A. flavus VCGs from agricultural produce is selectively adapted for invading crops under field condition.

We found that VCGs were strongly associated with mycotoxin and sclerotia production. Vegetatively compatible isolates shared the same mycotoxin combination and number of sclerotia per square centimeter of production. The only exception is isolate M15N3, which differed in mycotoxin production from the other isolates of the VCG A. It seems that there is a stronger association between belonging to a certain VCG and sclerotia production rather than mycotoxin production. VCGs already had been associated with many morphological and physiological features. However, association between mycotoxin combination and sclerotia production among isolates belonging to the same VCG is found for the first time. Papa (32) found no apparent relationship between VCGs and aflatoxin or sclerotium production. Bayman and Cotty (3) detected that, with the exception of one VCG, groups contained either $\mathrm{S}$ or $\mathrm{L}$ isolates. They also found significant differences in aflatoxin $\mathrm{B}_{1}$ production among VCGs, but they did not attempt to study aflatoxin $\mathrm{G}$ or CPA. Horn et al. (23) found positive correlation between total aflatoxin $\left(\mathrm{B}_{1}\right.$ and $\left.\mathrm{B}_{2}\right)$ and CPA in A. flavus. The number of sclerotia per plate was negatively correlated with kojic acid production and also detected that VCGs contained either S or L isolates.

Cotty (10) considered that differentiating strains by the occurrence of sclerotia is ambiguous and proposed that sclerotia size is important as a physiological trait. $\mathrm{He}$ stated that "isolates producing numerous small sclerotia were designated strain $\mathrm{S}$ ('small') isolates...isolates with any sclerotia greater than $400 \mu \mathrm{m}$ in diameter were designated strain L ('large') isolates". According to his criterion, all isolates used in our study belong to L strain. However, we observed substantial differences in sclerotia number and size of isolates between VCGs, particularly isolates included in VCGs C and E and the isolate M40N1, which also produced very small sclerotia and in larger quantities. We believe that these five isolates could correspond to the $\mathrm{S}$ strain, although all of them produced some sclerotia over $400 \mu \mathrm{m}$ in size (Table 2 ). The statistical analysis showed significant differences between isolates within the same VCG in sclerotia size but not in number of sclerotia per square centimeter. This suggests a better correlation between VCGs and the number of sclerotia than between VCGs and sclerotia size. Perhaps it would be better to consider the average size instead of the size of single sclerotia. Finally, we propose that it is not enough to differentiate $\mathrm{S}$ and $\mathrm{L}$ strain types by merely using sclerotia size, but that it is necessary to define a combination of traits that could take into account most of the diversity found.

Genetic diversity of a fungal population may be of major importance in developing a biocontrol strategy. Tran-Dinh et al. (35) suggested that, if genetic exchange can occur, the introduction of a more aggressive nontoxigenic isolate as a biocontrol agent could result in a recombinant with both increased aggressiveness and toxigenicity. In the present study, we found that aflatoxigenic and nonaflatoxigenic isolates grouped in different VCGs. Heterokaryon formation can only occur between vegetatively compatible isolates; therefore, these results enable the implementation of a biological control using a nonaflatoxigenic isolate belonging to a VCG different from the aflatoxigenic ones.

In populations of Aspergillus spp., each VCG may be a single clone; therefore, VCGs would be series of clones that were derived from a common ancestor (14). This idea of clonality has been supported by comparison of VCGs and restriction fragment length polymorphism of the mitochondrial genome (13) and analyses of random amplified polymorphic DNA (3). Information about the close relationships between isolates belonging to the same VCG is continuously increasing. In the present study, VCGs corresponded clearly with mycotoxin combination and sclerotia production by the isolates. Atypical isolates, simultaneously producing aflatoxins $\mathrm{B}, \mathrm{G}$, and CPA, grouped in a single VCG, indicating that the particular mycotoxin combination produced by these isolates could have arisen once in the region studied. It is necessary to continue the study of these atypical isolates, implementing new techniques to complement the information we have compiled. It would be of interest to study compatibility between our atypical isolates and those cited by Blaney et al. (4) from Australia. If these sets of isolates prove to be compatible with each other, we could propose a common origin, probably in the Southern Hemisphere.

The search for and study of new A. flavus isolates in Cordoba and other provinces is currently being conducted.

\section{ACKNOWLEDGMENTS}

We thank G. Vaamonde and others for making available the Aspergillus flavus and A. parasiticus isolates; J. E. Wright and G. Vaamonde for the critical review of the manuscript; and J. Calcagno for statistical advice.

\section{LITERATURE CITED}

1. Bayman, P., and Cotty, P. J. 1991. Vegetative compatibility and genetic diversity in the $A s$ pergillus flavus population of a single field. Can. J. Bot. 69:1707-1711.

2. Bayman, P., and Cotty, P. J. 1991. Improved media for selecting nitrate-nonuntilizing mutants in Aspergillus flavus. Mycologia 83:311316.

3. Bayman, P., and Cotty, P. J. 1993. Genetic diversity in Aspergillus flavus: association with aflatoxin production and morphology. Can. J. Bot. 71:23-31.

4. Blaney, B. J., Kelly, M. A., Tyler, A. L., and Connole, M. D. 1989. Aflatoxin and cyclopiazonic acid production by Queensland isolates of Aspergillus flavus and Aspergillus parasiticus. Aust. J. Agric. Res. 40:395-400.

5. Bosland, P. W., and Williams, P. H. 1987. An evaluation of Fusarium oxysporum from crucifers based on pathogenicity, isozyme polymorphism, vegetative compatibility, and geographic origin. Can. J. Bot. 65:2067-2073.

6. Brooker, N. L., Leslie, J. F., and Dickman, M B. 1991. Nitrate-nonutilizing mutants of Colletotrichum and their use in studies of vegetative compatibility and genetic relatedness. Phytopathology 81:672-677.

7. Correll, J. C., Gordon, T. R., and McCain, A H. 1988. Vegetative compatibility and pathogenicity of Verticillium albo-atrum. Phytopathology 78:1017-1021.

8. Correll, J. C., Klittich, C. J. R., and Leslie, J. F. 1987. Nitrate nonutilizing mutants of Fusarium oxysporum and their use in vegetative compatibility tests. Phytopathology 77:16401646.

9. Correll, J. C., Klittich, C. J. R., and Leslie, J. F. 1989. Heterokaryon self-incompatibility in Gibberella fujikuroi (Fusarium moniliforme). Mycol. Res. 93:21-27.

10. Cotty, P. J. 1989. Virulence and cultural characteristics of two Aspergillus flavus strains pathogenic on cotton. Phytopathology 79:808-814.

11. Cotty, P. J., and Bayman, P. 1993. Competitive exclusion of a toxigenic strain of Aspergillus flavus by an atoxigenic strain. Phytopathology 83:1283-1287.

12. Cove, D. J. 1976. Chlorate toxicity in Aspergillus nidulans: The selection and characterisation of chlorate resistant mutants. Heredity 36:191-203.

13. Croft, J. H. 1987. Genetic variation and evolution in Aspergillus. Pages 311-323 in: Evolutionary Biology of the Fungi. A. D. M Rayner, C. M. Brasier, and D. Moore, eds. Cambridge University Press, Cambridge.

14. Croft, J. H., and Jinks, J. L. 1977. Aspects of the population genetics of $A$. nidulans. Pages 339-360 in: Genetics and Physiology of Aspergillus. J. E. Smith and J. A. Pateman, eds. Academic Press, London.

15. Diener, U. L., Cole, R. J., Sanders, T. H., Payne, G. A., Lee, L. S., and Klich, M. A 1987. Epidemiology of aflatoxin formation by Aspergillus flavus. Annu. Rev. Phytopathol. 
25:249-270.

16. Diener, U. L., and Davis, N. D. 1966. Aflatoxin production by isolates of Aspergillus flavus. Phytopathology 56:1390-1393.

17. Dorner, J. W., Cole, R. J., and Diener, U. L. 1985. The toxicity of cyclopiazonic acid. Pages 529-535 in: Trichothecenes and Other Mycotoxins. J. Lacey, ed. John Wiley and Sons, Chichester, U.K

18. Egel, D. S., Cotty, P. J., and Elias, K. S. 1994. Relationships among isolates of Aspergillus sect. flavi that vary in aflatoxin production. Phytopathology 84:906-912.

19. Glass, N. L., and Kuldau, G. A. 1992. Mating type and vegetative incompatibility in filamentous Ascomycetes. Annu. Rev. Phytopathol. 30:201-224.

20. Gordon, T. R., and Okamoto, D. 1991. Vegetative compatibility groupings in a local population of Fusarium oxysporum. Can. J. Bot. 69:168-172

21. Hesseltine, C. W., Shotwell, O. L., Smith, M., Ellis, J. J., Vandegraft, E., and Shannon, G. 1970. Production of various aflatoxins by strains of the Aspergillus flavus series. Pages 202-210 in: Toxic Micro-organisms. M. Herzberg, ed. U. S. Gov. Print. Office, Washington, D.C.

22. Horn, B. W., and Greene, R. L. 1995. Vegetative compatibility within populations of Aspergillus flavus, A. parasiticus, and A. tamarii from a peanut field. Mycologia 87:324-332.

23. Horn, B. W., Greene, R. L., Sovalev, V. S.,
Dorner, J. W., and Powell, J. H. 1996. Association of morphology and mycotoxin production with vegetative compatibility groups in A. flavus, A. parasiticus and A. tamarii. Mycologia 88:574-587

24. Hyakumachi, M., and Ui, T. 1987. Non-selfanastomosing isolates of Rhizoctonia solani obtained from fields of sugar beet monoculture. Trans. Brit. Mycol. Soc. 89:155-159.

25. Jacobson, D. J., and Gordon, T. R. 1988. Vegetative compatibility and self-incompatibility within Fusarium oxysporum f. sp. melonis. Phytopathology 78:668-672.

26. Klich, M. A., and Pitt, J. I. 1988. Differentiation of Aspergillus flavus from A. parasiticus and other closely related species. Trans. Br. Mycol. Soc. 91:99-108.

27. Korolev, N., and Gindin, G. 1999. Vegetative compatibility in the entomopathogen Verticillium lecanii. Mycol. Res. 103:833-840.

28. Kurtzman, C. P, Horn, B. W., and Hesseltine, C. W. 1987. Aspergillus nomius, a new aflatoxin-producing species related to Aspergillus flavus and Aspergillus tamarii. Antonie van Leeuwenhoek J. Microbiol. Serol. 53:147158.

29. Kurtzman, C. P., Smiley, M. J., Robnett, C. J., and Wicklow, D. T. 1986. ADN relatedness among wild and domesticated species in the Aspergillus flavus group. Mycologia 78:955959.

30. Larkin, R. P, Hopkins, D. L., and Martin, F. N. 1990. Vegetative compatibility within Fusarium oxysporum f. sp. niveum and its relation- ship to virulence, aggressiveness, and race. Can. J. Microbiol. 36:352-358.

31. Leslie, J. F. 1993. Fungal vegetative compatibility. Annu. Rev. Phytopathol. 31:127-150.

32. Papa, K. E. 1986. Heterokaryon incompatibility in Aspergillus flavus. Mycologia 78:98101.

33. Pitt, J. I. 1993. Corrections to species names in physiological studies on Aspergillus flavus and Aspergillus parasiticus. J. Food Prot. 56:265-269.

34. Saito, M., Tsuruta, O., Siriacha, P., Kawasugi, S., Manabe, M., and Buangsuwon, D. 1986. Distribution and aflatoxin productivity of the atypical strains of Aspergillus flavus isolated from soils in Thailand. Proc. Jpn. Assoc. Mycotoxicol. 24:41-46.

35. Tran-Dinh, N., Pitt, J. I., and Carter, D. A 1999. Molecular genotype analysis of natura toxigenic and nontoxigenic isolates of Aspergillus flavus and A. parasiticus. Mycol. Res. 103:1485-1490.

36. Vaamonde, G., Degrossi, C., Comerio, R., and Fernández Pinto, V. Aspergillus flavus y $A$. parasiticus en maní cultivado en la provincia de Córdoba (Argentina): Características diferenciales y capacidad aflatoxicogénica. 1995 Bol. Soc. Arg. Bot. 30:191-198.

37. Vaamonde, G., Patriarca, A., Comerio, R., Vitale, R., and Fernández Pinto, V. 2000. Unusual pattern of mycotoxin production in Aspergillus section Flavi. Page 188 in: X International IUPAC Symposium on Mycotoxins and Phycotoxins, Guarujá, Brazil. 